\title{
Different Queuing Policies for Handover Requests in Low Earth Orbit Mobile Satellite Systems
}

\author{
Enrico Del Re, Senior Member, IEEE, Romano Fantacci, Senior Member, IEEE, \\ and Giovanni Giambene, Member, IEEE
}

\begin{abstract}
In this paper, a mobility model suitable for low earth orbit mobile satellite systems (LEO-MSS's) has been presented, and its statistical parameters have been derived in order to evaluate the impact of the mobility on the performance of the fixed channel allocation (FCA) strategy.

Moreover, we have foreseen that interbeam handover requests, which do not immediately find service, can be queued to reduce the handover failure rate. Two different queuing disciplines have been assumed: 1) the first-input-first-output (FIFO) scheme and 2) an idealized strategy that requires knowledge of the last useful instant (LUI) within which the handover procedure must be completed in order to rank the queued handover requests.

An analytical approach has been developed to compare these queuing techniques, and its results have been validated through simulations.
\end{abstract}

Index Terms-Mobile communications satellite systems, queuing theory.

\section{INTRODUCTION}

$\mathbf{F}$ UTURE global-coverage mobile satellite systems (MSS's) will be able to provide the users with communication services anytime and anywhere. MSS's will cover impervious or scarcely populated areas where the implementation of terrestrial cellular systems would be infeasible or too expensive. Moreover, in an integrated scenario, MSS's will also support terrestrial cellular networks when these will be congested or malfunctioning [1]-[3].

This paper is focused on low earth orbit (LEO) satellites [4]. The analysis here presented is kept general and illustrated numerically in the case of the IRIDIUM system [5].

Satellites are equipped with multispot-beam antennas that illuminate cells on the earth. A channel demand in a beam $x$ (i.e., a cell of the satellite system) may be due to either a new call arrival or a handover request from an adjacent beam (i.e., a mobile station that moves from a cell toward an adjacent cell); if no channel is available in $x$, the channel request fails and the relative user experiences the blocking of the call attempt or the dropping of a call in progress. From the user standpoint, it is more unacceptable the interruption of a conversation than the blocking of a newly arriving call.

In addition to this, interbeam handover requests are extremely frequent in LEO-MSS's during call lifetime and every time that a call has a beam change there is the risk that it

Manuscript received March 22, 1996; revised November 11, 1997. This work was supported by the Italian Space Agency and MURST.

The authors are with the Dipartimento di Ingegneria Elettronica, Università degli Studi di Firenze, 50139 Firenze, Italy.

Publisher Item Identifier S 0018-9545(99)00683-0. may be dropped owing to a lack of available resources in the destination cell of the mobile user.

Therefore, in future terrestrial microcellular and satellite systems (especially LEO-MSS's), techniques that prioritize the service of handover requests with respect to the service of new call attempts are deemed as essential in order to reduce as much as possible the call dropping probability and attain a satisfactory quality of service.

Many strategies have been recently proposed to privilege the handover service at the expenses of new call arrivals in case of terrestrial microcellular systems [6]-[9]. The aim of this paper is to study the performance of these policies in a satellite contest. In particular, a prioritization strategy based on the queuing of handovers, which do not immediately attain service, is considered here. In such a scheme, any handover request, which occurs in a cell where all channels are busy, can be queued for a maximum time $t_{w \max }$. Elapsed $t_{w \max }$, if no channel becomes available in the cell, and the call is still in progress, the handover procedure fails and the associated call is forced into termination.

In this study, a classical fixed channel allocation (FCA) technique has been assumed: that is, each cell has a predefined set of resources to satisfy the channel requests in it. Moreover, two different queuing disciplines for handover requests have been proposed and compared in terms of:

- the blocking probability for new call attempts $P_{b 1}$;

- the handover failure probability $P_{b 2}$;

- the call dropping probability $P_{\text {drop}}$;

- the probability $P_{n s}$ that a call is not completely served due to either the blocking of the call attempt or the failure of a subsequent handover request.

On the basis of ITU-T requirements for land mobile services [10], the values of $P_{\text {drop }}$ and $P_{b 1}$ should not exceed $5 \times 10^{-4}$ and $10^{-2}$, respectively. We consider that these requirements will be also valid for future high-quality MSS's.

An analytical approach has been developed to evaluate the performance of FCA with the capability of handover queuing. Analytical predictions have been validated by a comparison with simulation results.

In this paper, we refer to LEO-MSS's where the satellite antennas are not steered to point as long as possible the same region on the earth. Then, these LEO-MSS's are characterized by high time-varying conditions of the traffic offered to the cells, since LEO satellites (and then cells) move very fast with respect to the earth. In such a situation, the static frequency planning required by the FCA technique is not particularly 

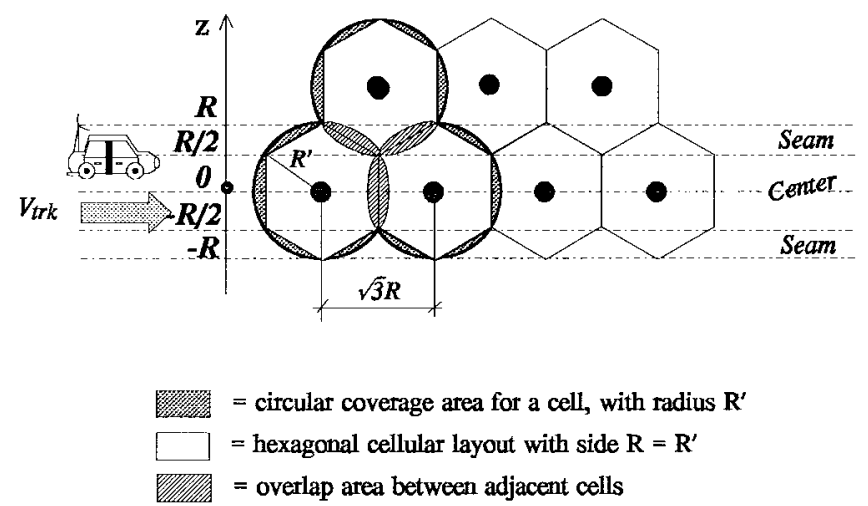

Fig. 1. The geometry assumed for the overlap areas (hexagonal cell side $=$ circular coverage radius).

efficient, even if it has been adopted for LEO-MSS's such as IRIDIUM and GLOBALSTAR. Another possible solution is represented by dynamic channel allocation [11], but the implementation of this technique is expensive in MSS's.

The organization of the remaining part of this paper is the following.

Section II deals with some preliminary assumptions used in this paper about MSS's. In Section III, the LEO mobility model is presented and analyzed. In Section IV, efficient handover strategies based on the queuing are considered. Section V presents the FCA technique. An analytical approach for evaluating the performance of the FCA technique with different queuing policies for handover requests is presented in Section VI. Finally, Section VII deals with simulation results and the comparison among the handover strategies proposed. The results for FCA without any prioritization strategy have been also shown for comparison purposes.

\section{PRELiminary CONSIDERATIONS AND ASSUMPTIONS ON LEO-MSS'S}

Let us assume that, due to beam-forming, spot-beam footprints are disposed on the earth according to a hexagonal regular layout (side $R$ ) and that they have a circular coverage with radius $R^{\prime}$. The possible values for the ratio $R^{\prime} / R$ range from 1 to 1.5 [12]. Obviously, the greater this ratio is, the larger the overlap area ${ }^{1}$ (between adjacent cells) is and then the better the queuing technique performance is. In this paper, the minimum possible extension for the overlap area has been considered (Fig. 1): $R=R^{\prime}$. In the numerical examples based on the IRIDIUM system, the value used for $R$ is purely indicative and it is an average between the minimum and the maximum values found in the literature, that is, $R=212.5 \mathrm{~km}$.

The cellular network irradiated on the earth by a multispotbeam antenna from a satellite has been considered parallelogram shaped. This network has been folded onto itself [13] in order to take into account the interference produced by the presence of adjacent spot beams belonging to neighboring satellites.

Due to the high value of the satellite ground-track speed, $V_{\text {trk }}$ (about $26000 \mathrm{~km} / \mathrm{h}$ in the LEO case), with respect to the

${ }^{1}$ An overlap area between two adjacent cells is a region where the MS can receive the signal from both cells.

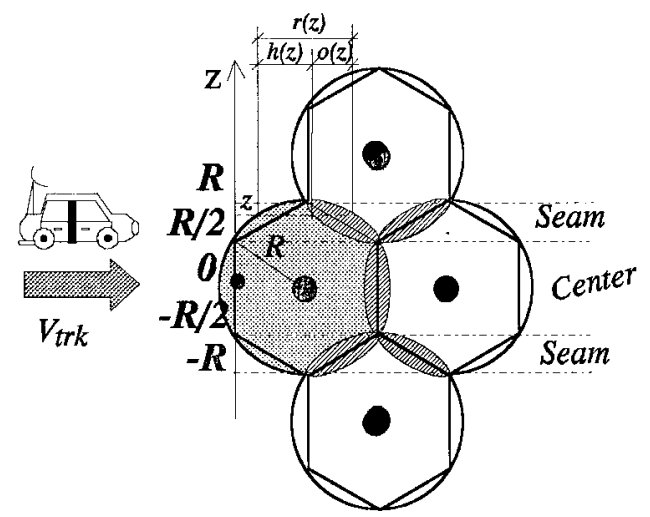

Fig. 2. The shape of the curvilinear cell and the distance crossed in the cell and in the overlap area for a given height $z$.

other motion component speeds (i.e., the earth rotation around its axis and the user motion relative to the earth), the relative satellite-user motion can be approximated by only vector $V_{\text {trk }}$; then, mobile stations (MS's) cross the cellular network irradiated by a satellite according to parallel straight lines.

Two different cells on the earth may use the same channel provided that they are at a suitable distance, called reuse distance $D$, which allows tolerable levels for the cochannel interference. Along this paper, it will be assumed that $D=\sqrt{21} R$. Here, we do not consider the physical nature of communication channels; they are simply considered as resources shared among users according to a set of rules. In this work, an FCA technique has been considered, as it will be explained in Section V.

Only voice traffic has been considered; as in the classical fixed telephony, new call attempts that do not immediately find available resources are blocked and lost. Moreover, only mobile-to-fixed users calls and fixed-to-mobile users calls have been taken into account, because mobile-to-mobile users calls are expected to be a little percentage of the whole traffic (max. $5 \%)$.

\section{THE LEO MOBILITY MODEL}

In what follows, source cell denotes the cell where the MS call starts and transit cell denotes any subsequent cell reached by the MS with the call in progress. Let us consider an MS that crosses a cell at a height $z \in[R, R]$ (Fig. 1). Let $r(z)$ denote the length of the circular cell with radius $R$ at height $z$ (see Fig. 2)

$$
r(z)=2 \sqrt{R^{2}-z^{2}}
$$

The circular cell is divided into two regions: 1) the overlap area with adjacent cells in the direction of the satellite-user relative motion and 2) the remaining part of the cell that is called curvilinear cell. The curvilinear cell (whose area is equal to $3 \sqrt{3} R^{2} / 2$ ) is not hexagonal, but it is represented by the shaded area in Fig. 2. We have denoted by $h(z)$ the distance crossed by the MS in the curvilinear cell at a height $z$, and we have denoted by $o(z)$ the relevant distance covered in the overlap area

$$
h(z)=r(z)-o(z)
$$


and

$$
o(z)=\left\{\begin{array}{cl}
2 \sqrt{R^{2}-z^{2}}-\sqrt{3} R, & \text { if }|z| \leq \frac{R}{2} \\
\sqrt{R^{2}-z^{2}}-\frac{\sqrt{3}}{2} R+\sqrt{R^{2}-\left(|z|=\frac{3}{2} R\right)^{2}}, \\
\text { if } R \geq|z|>\frac{R}{2} .
\end{array}\right.
$$

In the following part of this paper, the curvilinear cell (resulting from the assumptions on both mobility and cellular layout) will be simply called cell.

Once the position of the MS at the call arrival instant is defined, a height $z$ is assigned to this call in its source cell. Then, the distance $o(z)$ covered in the overlap area is obtained from (3) and, due to the geometry of the problem, this value is valid for any subsequent handover request, it does not matter if it originates from a source cell or a transit one.

The LEO mobility model proposed in this paper can be summarized as follows.

1) MS's cross the cellular network with a relative velocity, vector $V_{\text {trk }}$ "orthogonal" to the side of the cells (see Fig. 1).

2) When a handover occurs, the destination cell is the neighboring cell in the direction of the relative satelliteuser motion.

3) Calls are uniformly generated all over the network.

4) From the call outset in a cell, the related MS travels a distance (depending on $z$ ) defined as:

a. uniformly distributed between zero and $h(z)$ if the cell is the source cell of the call;

b. deterministically equal to $h(z)$ if the cell is a transit cell of the call.

According to the third assumption, the probability density function (pdf) of the height $z$ of a call arrival in a cell is $f_{i}(z)$, where $i=1$ for a call in its source cell and $i=2$ for a call in a transit cell

$$
f_{i}(z)= \begin{cases}\frac{h(z)}{3 \sqrt{3}}, & \text { if } i=1 \\ \frac{\{u[z+R]-u[z-R]\}}{2 R}, & \text { if } i=2\end{cases}
$$

where

$$
u(x)= \begin{cases}1, & \text { for } x>0 \\ 0, & \text { otherwise. }\end{cases}
$$

We introduce the following dimensionless parameter that characterizes the MS mobility in a cell, according to the height $z:$

$$
\alpha(z)=\frac{h(z)}{V_{\mathrm{trk}} T_{m}}
$$

where $T_{m}$ is the average call duration.

For $|z| \leq R / 2, h(z)=\sqrt{3} R$ and $\alpha(z)$ is constant and equal to $\sqrt{3} R /\left(V_{\mathrm{trk}} T_{m}\right)$; this quantity will be denoted by $\alpha$. For the IRIDIUM satellite constellation $(R=212.5 \mathrm{~km}$, $\left.V_{\mathrm{trk}}=26600 \mathrm{~km} / \mathrm{h}, T_{m}=3 \mathrm{~min}\right), \alpha$ is about equal to 0.27 .
The unencumbered call duration $t_{d}$ has been assumed a random variable exponentially distributed with mean $T_{m}$. Let $t_{m c 1}$ be the time interval elapsed from the arrival instant of a new call in its source cell to the instant in which the relative MS reaches the borders of an adjacent cell. Let $t_{m c 2}$ be the time interval from the handover request instant toward a transit cell to the instant in which the relative MS reaches the borders of an adjacent cell.

Let us note the following [14].

- The handover probability for a call from a cell, where the related MS crosses (from the call arrival instant) a distance uniformly distributed between zero and $x V_{\mathrm{trk}} T_{m}$ is $P_{h 1}(x)$ given by

$$
P_{h 1}(x)=\frac{1-e^{-x}}{x}
$$

- The handover probability for a call from a cell, where the related MS crosses (from the call arrival instant) a fixed distance equal to $x V_{\mathrm{trk}} T_{m}$ is $P_{h 2}(x)$ given by

$$
P_{h 2}(x)=e^{-x} \text {. }
$$

Then, handover probabilities $P_{H 1}$ and $P_{H 2}$, respectively, from the source cell and a transit one are obtained according to the following formula:

$$
P_{H i}=\int_{-R}^{R} P_{h i}(\alpha(z)) f_{i}(z) d z .
$$

From (9), with $i=1$, the handover probability from a source cell is

$$
P_{H 1}(\alpha)=\frac{2}{3} P_{h 1}(\alpha)+\frac{\frac{4}{3 R} \int_{R / 2}^{R} \alpha(z) P_{h 1}(\alpha(z)) d z}{\alpha} .
$$

From (9), with $i=2$, the handover probability from a transit cell is

$$
P_{H 2}(\alpha)=\frac{P_{h 2}(\alpha)}{2}+\frac{1}{R} \int_{R / 2}^{R} P_{h 2}(\alpha(z)) d z .
$$

The integral functions in (10) and (11) cannot be expressed in terms of elementary functions and must be numerically evaluated.

Handover probabilities $P_{H 1}$ and $P_{H 2}$ only depend on the mobility parameter $\alpha$. It is evident that as $\alpha$ approaches $0(\infty)$, $P_{H 1}$ and $P_{H 2}$ approach 1 (0), i.e., the mobility increases (decreases). In particular, in the IRIDIUM case, $P_{H 1} \approx 89 \%$ and $P_{H 2} \approx 81 \%$.

The channel holding time in a cell can be derived as

$$
t_{H i}=\min \left[t_{d}, t_{m c i}\right]
$$

where $i=1$ for a call in its source cell and $i=2$ for a call in a transit cell.

From [15], the expected value of $t_{H i}, E\left[t_{H i}\right]$ results in

$$
E\left[t_{H i}\right]=T_{m}\left(1-P_{H i}\right) .
$$

Let us consider an MS that starts a call in the source cell at a height $z \in[R, R]$ assumed as a random variable with probability distribution function $f_{1}(z)$ given by (4). 


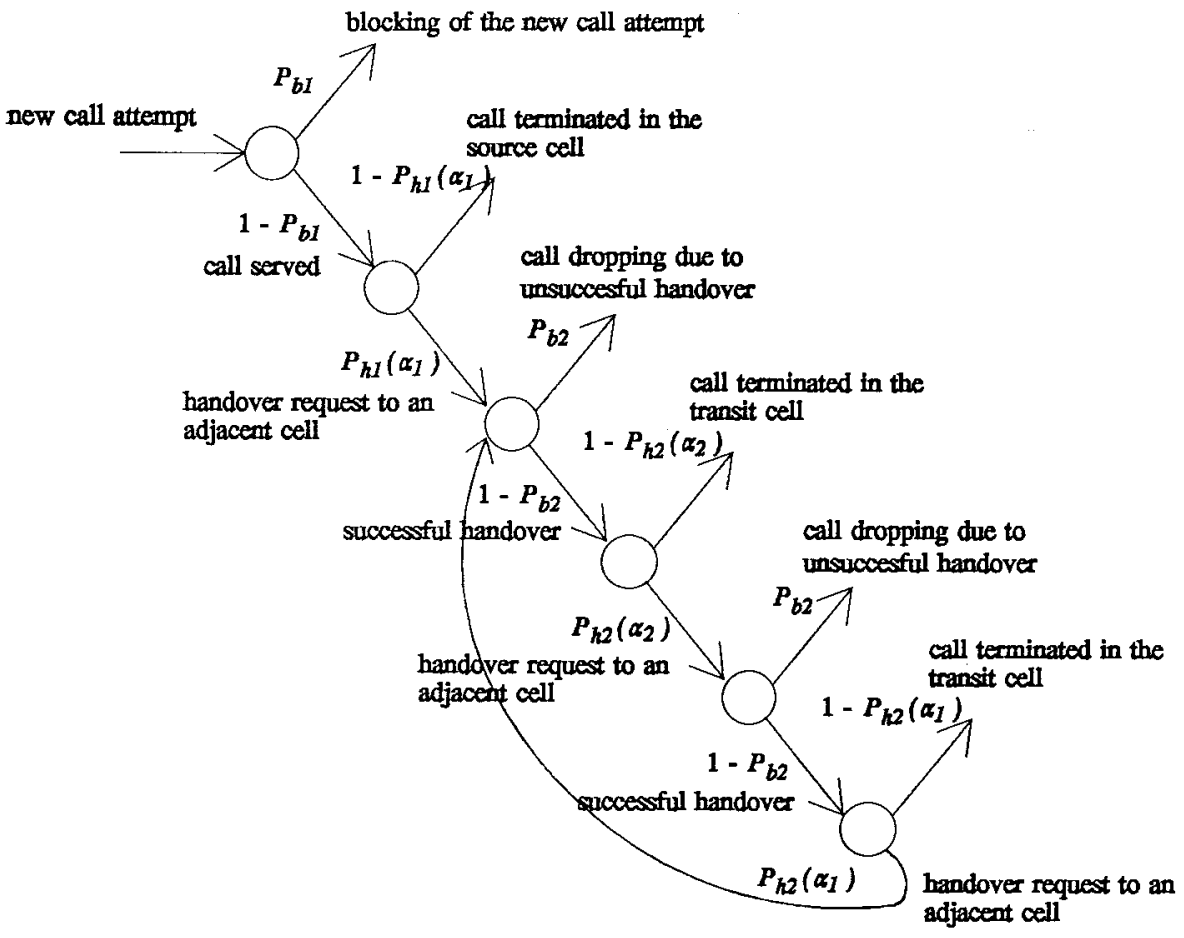

Fig. 3. The handover process across the seam of the cellular network.

If $|z| \leq R / 2$ (see Fig. 1), the MS motion is centered with respect to the cellular network. In such a case, $P_{h 1}(\alpha)$ is the handover probability from the source cell [see (7)] and $P_{h 2}(\alpha)$ is the handover probability from a transit cell [see (8)] [14]. Then, we can use the result already obtained in [14] in order to express the mean number of handover requests per call for $|z| \leq R / 2$

$$
n_{h \mid z}=\frac{P_{h 1}(\alpha)\left(1-P_{b 1}\right)}{1-\left(1-P_{b 2}\right) P_{h 2}(\alpha)}, \quad \text { for }|z| \leq \frac{R}{2} .
$$

If $R \geq|z|>R / 2$ (see Fig. 1), the MS moves along the seam of the cellular network; the maximum distance covered in the source cell ${ }^{2}$ is $h(z)$, whereas the distance covered in the subsequent transit cell ${ }^{2}$ (if the MS has a call still in progress) is $\sqrt{3} R-h(z)$. In the next transit cell (if the MS has a call still in progress), the distance crossed by the $\mathrm{MS}^{2}$ is again $h(z)$ and so on alternately. Let us denote

$$
\begin{aligned}
& \alpha_{1}(z)=\frac{h(z)}{V_{\text {trk }} T_{m}} \quad \alpha_{2}(z)=\frac{\sqrt{3}-h(z)}{V_{\text {trk }} T_{m}} \\
& \text { then } \quad \alpha_{1}(z)+\alpha_{2}(z)=\alpha .
\end{aligned}
$$

In a source cell, the distance covered by the $\mathrm{MS}^{2}$ is uniformly distributed between zero and $h(z)$, therefore, the handover probability is given by $P_{h 1}\left(\alpha_{1}\right)$ [14]; in the subsequent transit cell (if the call is still in progress), the distance covered ${ }^{2}$ is deterministically equal to $\sqrt{3} R-h(z)$, therefore, the handover probability is given by $P_{h 2}\left(\alpha_{2}\right)$ [14]. This leads to the handover process shown in Fig. 3. On the basis of this diagram,

\footnotetext{
${ }^{2}$ It has been considered the distance crossed in a cell from the call arrival instant in it to the instant when the borders with an adjacent cell are reached by the relevant MS.
}

the distribution of the number of handover requests per call $h_{n}$ has been derived and shown in Table I.

The average value of $h_{n}$ for $R \geq|z|>R / 2$ is given by

$$
\begin{aligned}
& n_{h \mid z}=\left(1-P_{b 1}\right) \frac{P_{h 1}\left(\alpha_{1}\right)+\left(1-P_{b 2}\right) P_{h 1}\left(\alpha_{1}\right) P_{h 2}\left(\alpha_{2}\right)}{1-\left(1-P_{b 2}\right)^{2} P_{h 2}(\alpha)}, \\
& \quad \text { for } R \geq|z|>\frac{R}{2} .
\end{aligned}
$$

The average number of handover requests per call $n_{h}$ can be obtained by removing the conditioning on $z$ in formulas (14) and (16); it is necessary to integrate $n_{h \mid z}$ weighted by the pdf of $z$, i.e., $f_{1}(z)$, from $-R$ to $R$. We have obtained the following result:

$$
\begin{aligned}
n_{h}= & \left(1-P_{b 1}\right) \frac{2}{3}\left\{\frac{P_{h 1}(\alpha)}{1-\left(1-P_{b 2}\right) P_{h 2}(\alpha)}\right. \\
& \left.+\frac{\gamma(\dot{\alpha})+\left(1-P_{b 2}\right) \zeta(\alpha)}{1-\left(1-P_{b 2}\right)^{2} P_{h 2}(\alpha)}\right\} \quad \frac{\text { handovers }}{\text { call }}
\end{aligned}
$$

where

$$
\begin{aligned}
\zeta(\alpha) & =\frac{2}{\alpha}\left(P_{H 2}(\alpha)-P_{h 2}(\alpha)\right) \\
\gamma(\alpha) & =P_{h 1}(\alpha)-\zeta(\alpha) .
\end{aligned}
$$

From (17), we can note that if $P_{b 1}=P_{b 2}=0, n_{h}=4 /(3 \alpha)$; in this case, parameter $n_{h}$ only depends on network topology. For the IRIDIUM system, $a \approx 0.27$ and $n_{h} \approx 4.82$ handovers/call, when $P_{b 1}=P_{b 2}=0$.

The average number of handover requests per call accepted into the network $n_{h}^{\prime}$ can be obtained from $n_{h}$ through the following formula (see Fig. 3):

$$
n_{h}^{\prime}=\frac{n_{h}}{1-P_{b 1}} .
$$


TABLE I

The Distribution of VARiable $h_{n}$

\begin{tabular}{|c|c|}
\hline, $\mathbf{l}_{n}$ & Probability \\
\hline 0 & $1-P_{h l}\left(\alpha_{1}\right)\left(1-P_{b l}\right)$ \\
\hline 1 & $\left(1-P_{b i}\right) P_{h l}\left(\alpha_{1}\right)\left[1-P_{h 2}\left(\alpha_{2}\right)\left(1-P_{b 2}\right)\right]$ \\
\hline 2 & $\left(1-P_{b 1}\right) P_{h 1}\left(\alpha_{t}\right) P_{h 2}\left(\alpha_{2}\right)\left(1-P_{b 2}\right)\left[1-P_{h 2}\left(\alpha_{l}\right)\left(1-P_{b 2}\right)\right]$ \\
\hline 3 & $\left(1-P_{b 1}\right) P_{h 1}\left(\alpha_{1}\right) P_{h 2}(\alpha)\left(1-P_{b 2}\right)^{2}\left[1-P_{h 2}\left(\alpha_{2}\right)\left(1-P_{b 2}\right)\right]$ \\
\hline .......... & ......... \\
\hline $2 k$ & $\left(1-P_{b 2}\right) P_{h l}\left(\alpha_{1}\right)\left[P_{h 2}(\alpha)\right]^{k-1} P_{h 3}\left(\alpha_{2}\right)\left(1-P_{b 2}\right)^{2 t-1}\left[1-P_{h}\left(\alpha_{2}\right)\left(1-P_{b 2}\right)\right]$ \\
\hline $2 k+1$ & $\left(1-P_{b i}\right) P_{h 1}\left(\alpha_{1}\right)\left[P_{h 2}(\alpha)\right]^{k}\left(1-P_{b 2}\right)^{2 k}\left[1-P_{h 2}\left(\alpha_{2}\right)\left(1-P_{b 2}\right)\right]$ \\
\hline
\end{tabular}

It is easy to note that if a served call originates an average number $n_{h}^{\prime}$ of handover requests and at each request the call may be dropped with probability $P_{b 2}$, then the overall call dropping probability is given by

$$
P_{\text {drop }}=n_{h}^{\prime} P_{b 2} \text {. }
$$

Probability $P_{n s}$ can be obtained as follows [8], [14]:

$$
P_{n s}=P_{b 1}+\left(1-P_{b 1}\right) P_{\text {drop }}=P_{b 1}+n_{h} P_{b 2} \text {. }
$$

Let us define parameter $n_{h}^{\circ}$ that represents the average number of successful handovers per call accepted into the network. The following formula is valid for $n_{h}^{\circ}$ :

$$
n_{h}^{\circ}=n_{h}^{\prime}-P_{\text {drop }}
$$

Let us assume a uniform traffic: $\lambda_{h}$ denotes the average arrival rate of handover requests toward a generic cell and $\lambda$ denotes the average arrival rate of new call attempts in a generic cell, so we have

$$
n_{h}=\frac{\lambda_{h}}{\lambda} .
$$

Equation (24) is general and valid for any mobility model.

\section{Handover Queuing Policy}

The performance of a channel allocation technique is strongly dependent on the handover management strategy; in order to meet the specified requirements for the call dropping probability, an interbeam handover strategy is here considered that requires the queuing of handover $(\mathrm{QH})$ requests when no channel is available in the destination cell of the relevant MS at the handover request instant.

\section{A. The Description of the Queuing Technique}

Let us assume that an active MS moves from cell $x$ toward an adjacent cell $y$; the competent satellite recognizes the handover need as soon as the level of the signal it receives from the MS drops below a suitable threshold (networkcontrolled handover) [16]. In this paper, we consider that this event corresponds to the instant when the MS enters the overlap area between cell $x$ and cell $y$. A network-controlled handover procedure has the advantage of a reduced radio link signaling load and a low MS complexity, therefore, it can be easily implemented in a satellite system.

The MS crosses the overlap area in a time $t_{w \max }$. In cell $y$, the MS must be provided with a new channel to carry on the communication. If no channel is immediately available in cell $y$, the handover request can be queued for a maximum time $t_{w \max }$, waiting for a free resource in $y$. Let $\Lambda(j)$ denote the number of free channels in the generic cell $j$. An interbeam handover request is served according to the following steps.

1) If it results $\Lambda(y) \neq \emptyset$, the handover is immediately served in cell $y$ and a call termination is performed in cell $x$.

2) If it results $\Lambda(y)=\emptyset$, the handover request is queued waiting for an available channel in cell $y$. In the meantime, the call is served by the originating cell. A handover request leaves the queue owing to one of the three following reasons.

a) The handover procedure is successful: The handover request is served, before the call is ended and its maximum queuing time has expired.

b) The handover procedure has been useless: The call ends before the corresponding handover request is served and its maximum queuing time has expired.

c) The handover procedure fails and the call is dropped: The handover has not been performed within $t_{w \text { max }}$ and the call is not ended before its maximum queuing time has expired.

\section{B. Statistics of the Maximum Queuing Time}

According to the basic assumptions (see Section II) and the mobility model (see Section III), the randomness of $t_{w \max }$ only depends on the height $z$ that is related to the call in its source cell; in particular, $t_{w \max }$ is derived as the time spent by the associated MS to cross the overlap area at a given height $z$ (see Fig. 1) with a speed $V_{\text {trk }}$

$$
t_{w \max }=\frac{o(z)}{V_{\mathrm{trk}}} .
$$


The average value of $o(z)$ has been found according to the following formula:

$$
E[o(z)]=\int_{-R}^{+R} o(z) f_{1}(z) d z=\sqrt{3} R \beta
$$

where

$$
\beta=\frac{4}{9}\left(\frac{\sqrt{3}}{3} \pi-\frac{3}{2}\right) \approx 0.1394
$$

and $\beta$ is a dimensionless parameter that only depends on the geometric assumptions for both the mobility model and the overlap areas.

Accordingly, the average value of the maximum queuing time $E\left[t_{w \max }\right]$ results in

$$
E\left[t_{w \max }\right]=\frac{E[o(z)]}{V_{\mathrm{trk}}}=\alpha T_{m} \beta
$$

In particular, in the IRIDIUM case we have:

$$
\text { - } E[o(z)] \approx 52 \mathrm{~km}
$$$$
\text { - } E\left[t_{w \max }\right] \approx 7 \mathrm{~s} \text {. }
$$

\section{Queuing Disciplines}

Two main approaches are possible to order the handover requests in the queue: the first-input-first-output (FIFO) and the measurement-based prioritization scheme (MBPS).

In the FIFO scheme [13], [14], handover requests are queued according to their arrival instants, whereas the MBPS scheme [7], [17]-[19] uses a nonpreemptive dynamic priority to establish the ranking of the handover requests in the queue; the priorities are defined by power level measurements on the signal received by the satellite. The target is to serve first the request from the MS with a more degraded link. The quality of the link is continuously monitored for each handover request in the queue in order to update its position. This policy manages handover requests in a better way than the FIFO one, but it requires a greater implementation complexity.

Now, let us consider an idealized queuing scheme that will be denoted by last useful instant (LUI). This new strategy relies on the fact that when a handover request is queued, the system exactly knows its maximum queuing time $\left(t_{w \max }\right)$. This request is placed before the other handover requests found in the queue that have a greater residual maximum queuing time. Then, the system serves first the most critical handover request. This queuing policy is not dynamic: the relative ranking of queued handover requests does not change while they are waiting for service.

In the LEO-MSS's under examination, it is possible to consider an implementation of the LUI strategy, since the mobility is dominated by the satellite motion. In particular, the system may evaluate (with a sufficient accuracy) the time $t_{w \max }$ for a queued handover request as follows: 1) the MS position is determined at the beginning of the call, as described below; 2) the MS position can be easily tracked during the call $^{3}$; 3) the time $t_{w \max }$ can be obtained by using $(25),{ }^{4}$ since the system knows both the MS motion and the cellular coverage geometry and then can estimate the distance crossed in the overlap area. The efficiency of this version of the LUI scheme suitable for implementation in LEO-MSS's depends on the accuracy of the MS position estimation.

As for the positioning system, we consider a solution integrated into the LEO-MSS itself: the MS position can be estimated by the LEO-MSS by measuring the propagation delay and Doppler frequency shift for the MS transmissions [20]. Using the time delay measurement, a fixed propagation delay circle is obtained on the earth. Since the Doppler frequency shift is related to the angle between the satellite velocity vector and the direction vector from the MS to the satellite, the Doppler measurement defines a cone making a fixed angle with the satellite velocity vector. The intersection on the earth between the constant propagation delay circle and the cone identifies two points. A possible solution to solve this spatial ambiguity is to take another Doppler frequency shift measurement from a second satellite in visibility (this solution adds some constraints on the satellite constellation). For more details, [20] and [21] can be considered.

The LUI strategy version suitable for implementation in LEO-MSS's is similar to the MBPS scheme, but the handover ordering is based on MS position estimations rather than on power level measurements. In the following sections the performance analysis will be carried out by assuming an ideal LUI scheme, where the system knows the exact value of $t_{w \max }$ for each handover request.

\section{The FCA TeChNique}

With FCA, a set of channels is permanently assigned to each cell, according to the allowed reuse distance $D$. A call can only be served by an available channel (if any) belonging to the set of the cell. If a channel request does not find any free nominal channel in the cell, the call is blocked and lost. For uniform traffic offered to the cells, the entire pool of $M$ channels at disposition of the system is divided into equal groups each one composed by $S$ channels [22]

$$
S=\frac{M}{K}, \quad \text { where } \quad K=\frac{D^{2}}{3 R^{2}} .
$$

In the following, FCA (or, equivalently, FCA NPS) will denote the FCA with no prioritization scheme (NPS) for handover requests, whereas FCA-QH will denote the FCA with the queuing of handover requests.

\footnotetext{
${ }^{3}$ Once a first MS position measurement has been taken, the MS position can be tracked by estimating position variations on the basis of satellite ephemerides (this is possible because, in the LEO case, the relative satelliteMS motion is dominated by the satellite motion).

${ }^{4}$ Of course, $t_{w \max }$ depends not only on geometrical considerations, but also on propagation aspects. However, since LEO-MSS's are expected to serve mobile uses with sufficiently high elevation angles, we may neglect, with a good approximation, propagation aspects in deriving $t_{w \max }$.
} 


\section{ANALYSIS OF FCA-QH WITH DiFFERENT QUEUING STRATEGIES}

In this section, an analytical approach for evaluating the FCA-QH performance is presented. In performing our analysis, we have assumed the following.

- $S$ channels are assigned per cell according to (29).

- New call arrivals and handover attempts are two independent Poisson processes, with mean rates $\lambda$ and $\lambda_{h}$, with $\lambda_{h}$ related to $\lambda$ by (17) and (24).

- Whether handover requests are queued or not, the channel holding time in a cell (for both new call arrivals and handovers) is approximated by a random variable with an exponential distribution and mean $1 / \mu$ given by

$$
\begin{aligned}
\frac{1}{\mu}= & \frac{\lambda\left(1-P_{b 1}\right)}{\lambda\left(1-P_{b 1}\right)+\lambda_{h}\left(1-P_{b 2}\right)} E\left[t_{H 1}\right. \\
& +\frac{\lambda_{h}\left(1-P_{b 2}\right)}{\lambda\left(1-P_{b 1}\right)+\lambda_{h}\left(1-P_{b 2}\right)} E\left[t_{H 2}\right] \\
= & \frac{\left.E_{[} t_{H 1}\right]+n_{h}^{\circ} E\left[t_{H 2}\right]}{1+n_{h}^{\circ}}
\end{aligned}
$$

where $E\left[t_{H 1}\right]$ and $E\left[t_{H 2}\right]$ are derived from (13) and $n_{h}^{\circ}$ is given by (23).

- The maximum waiting time is approximated by a random variable exponentially distributed, with expected value equal to $1 / \mu_{w}=E\left[t_{w \max }\right]$, where $E\left[t_{w \max }\right]$ is given by (28).

- The handover queue has an infinite capacity.

It is important to note that on the basis of the cellular layout and the mobility model, respectively described in Sections II and III, the distributions for both the time spent by an MS in the overlap area and the channel holding time in a cell are not exponential. In both cases, the previously listed assumptions consider fitted exponential distributions with the same expected value of the relevant actual distributions [i.e., (28) and (30), respectively]. The goodness of these assumptions has been verified through the evaluation of parameter $G$ defined by Hong and Rappaport in [8]. Parameter $G \in[0,1]$ measures the degree of fitting between two distributions through a normalized integral difference between their complementary functions. Note that a value of $G$ close to zero denotes a good fitting. It has been verified that the exponential distributions with the same expected values of the actual distributions attain a very good fitting (in both cases, $G$ is quite little: $G \approx 0.17$ ) very close to the optimum; the exponential distributions which minimize $G$ give $G \approx 0.16+0.17$ in both cases with slightly different expected values as regards those in (28) and (30), but these differences have no practical impact on the estimation of the blocking performance. Therefore, for the sake of simplicity, exponential distributions with the expected values given by (28) and (30) will be used in this section for performance analysis.

Another approximation in the above list of assumptions concerns the Poisson arrival process for handover requests in a cell because this is a smooth traffic [23]. In order to demonstrate this point, let us consider a loss queuing system of the $M / M / S / S$ type ( $M$ : Poisson arrival process $M$ : exponentially distributed service time/ $S$ : number of servers $/ S$ :

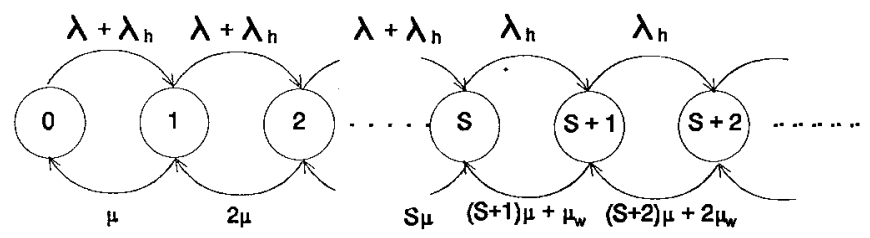

Fig. 4. The queuing system for FCA-QH.

number of requests in the system); the output traffic from this system is not Poisson, but it is smooth, whereas the overflow traffic is peaked [23]. A smooth traffic gives a lower blocking probability than a Poisson one at a parity of system resources, average arrival rate, service time distribution. Then, for the sake of simplicity, let us refer to the LEO-MSS with FCA: each cell can be modeled as a loss queuing system. Even if the arrival process for new call attempts in a cell is Poisson, the handover arrival process is not Poisson because it can be thought as the output traffic from the loss queuing system that models the cells from which the handover request may be originated. Analogous considerations can be made for LEO-MSS's with FCA-QH. Accordingly, the handover arrival process is a smooth traffic and this characteristic is magnified in LEO-MSS's because handover requests are highly frequent during call lifetime. Therefore, the analysis based on the Poisson arrival process for handovers permits to attain a conservative estimate of system performance.

Other analytical studies presented in the literature [7], [8], [17] do not consider the possibility that a call, that originates a handover request which is queued, may end before obtaining service in the destination cell of the MS and before its maximum queuing time has expired. This study removes such approximation, ${ }^{5}$ as it will be explained in the following analysis.

From the above assumptions, it follows that in the case of the FCA-QH technique each cell can be modeled as an $M / M / S$ queuing system with nonhomogeneous arrival rates [8], [14] ( $M$ : Poisson arrival process $/ M$ : service time exponentially distributed $/ S$ : number of channels assigned per cell). The queuing model has been shown in Fig. 4.

The state of the queuing system under consideration (i.e., a cell of the network) has been defined as sum of the number of calls in service and the number of queued handovers. Whenever the system is in a state $n$ less than $S$, the gross arrival rate is $\lambda+\lambda_{h}$; while if the state is greater or equal to $S$, i.e., all channels are busy, the gross arrival rate is $\lambda_{h}$ (Fig. 4).

When the system is in the state $S+i$, for $i=1,2, \cdots$, we have considered the following contributions to the departure rate (Fig. 4):

- $S_{m}$ due to the service completion for a request in the queue;

- $i \mu_{w}$ since a queued handover request may be cleared before attaining service;

- $i \mu$ because a call may end in the overlap area before obtaining service (this contribution gives rise to a different analysis with respect to that outlined in [14]).

\footnotetext{
${ }^{5}$ Note that the smaller $\left[E_{t \max }\right] / T_{m}=\alpha \beta$ is, the better the approximation that neglects the calls ended in the overlap area is.
} 
The model shown in Fig. 4 is valid for both FIFO and LUI queuing disciplines; what changes in both cases is the derivation of the handover failure probability $P_{b 2}$ according to the different ways for managing handover requests.

Let us analyze the state probabilities for the Markov chain in Fig. 4; by following the same approach proposed in [14], the probability of the state $n, P_{n}$ can be derived as

$$
P_{n}= \begin{cases}\frac{\left(\lambda+\lambda_{h}\right)^{n}}{n ! \mu^{n}} P_{0}, & 1 \leq n \leq S-1 \\ \frac{\left(\lambda+\lambda_{h}\right)^{S} \lambda_{h}^{n-S}}{n-S} P_{0}, & n \geq S \\ S ! \mu^{S} \prod_{j=1}^{n}\left((S+j) \mu+j \mu_{w}\right) & \end{cases}
$$

where the idle system probability $P_{0}$ is given by

$$
\begin{aligned}
P_{0}= & \left\{\sum_{n=0}^{S-1}\left[\frac{\left(\lambda+\lambda_{h}\right)^{n}}{n ! \mu^{n}}\right]+\sum_{n=S}^{\infty}\right. \\
& \left.\cdot\left[\frac{\left(\lambda+\lambda_{h}\right)^{S} \lambda_{h}^{n-S}}{S ! \mu^{S} \prod_{j=1}^{n-S}\left((S+j) \mu+j \mu_{w}\right)}\right]\right\}^{-1} .
\end{aligned}
$$

New arrivals are blocked when all the available channels (servers) are in use in the cell, i.e., when the queuing system is in the state $n \geq S$. Therefore, $P_{b 1}$ results in

$$
P_{b 1}=\sum_{n=S}^{\infty} P_{n}
$$

\section{A. $P_{b 2}$ with FIFO Queuing Policy}

In this case $P_{b 2}$ can be derived by following the same approach proposed in [8], [14] and by taking account of these new aspects.

1) $P_{b 2}$ must contain a multiplying factor $P_{u h}=\mu_{w} /(\mu+$ $\left.\mu_{w}\right)$ which represents the probability that the queued handover request is related to a call that does not end before its time $t_{w \max }$ has expired.

2) State probabilities are derived according to the new queuing model (Fig. 4), as shown in (31) and (32).

3) We take account of the additional departure rates $i \mu$ for states $S+i$ with $i=1,2, \cdots$ that have been introduced to consider the handover requests whose calls end in the overlap area before obtaining service and before their maximum queuing times have expired.

$P_{b 2}$ results in

$$
\begin{aligned}
P_{b 2}= & \frac{\mu_{w}}{\mu+\mu_{w}} \sum_{n=S}^{\infty} P_{n}\left\{1-\frac{S \mu}{S \mu+\mu_{w}}\right. \\
& \left.\cdot \prod_{j=1}^{n-S}\left[1-\frac{\mu_{w}}{S \mu+\mu_{w}}\left(\frac{\mu_{w}}{\mu+\mu_{w}} \frac{1}{2}\right)^{j}\right]\right\} .
\end{aligned}
$$

\section{B. $P_{b 2}$ with LUI Queuing Policy}

In the case of the LUI strategy, each handover request in the queue reaches anyhow the head of the queue (unless the request leaves the queue because the call is ended) before dropping the associated call. Only the call related to the handover at the head of the queue may be dropped. This entails that the failure probability for a handover request that has been queued since it did not find any free resource among the $S, P_{b 2 \mid S}$ does not depend on the position that this request initially had in the queue. Then, by using (33), the following result is valid:

$$
P_{b 2}=\sum_{n=S}^{\infty} P_{n} P_{b 2 \mid S}=P_{b 2 \mid S} P_{b 1}
$$

where $P_{b 2 \mid S}$ takes into account two joined and independent events.

- The call related to the handover request at the head of the queue does not end before leaving the overlap area; the probability of this event is $P_{u h}$ that has been already used to derive $P_{b 2}$ in the FIFO case.

- None of the $S$ channels of the cell becomes free before the maximum queuing time has expired; let us denote the probability of this event by $P_{f}$.

By using the fitted exponential distributions for both the maximum queuing time (expected value $=1 / \mu_{w}$ ) and the channel holding time (expected value $=1 / \mu$ ), we obtain through some algebraic computations

$$
P_{u h}=\frac{\mu_{w}}{\mu+\mu_{w}} \quad P_{f}=\frac{\mu_{w}}{S \mu+\mu_{w}} .
$$

Hence, in the LUI case, $P_{b 2}$ is given by

$$
P_{b 2}=P_{b 1} \frac{\mu_{w}}{\mu+\mu_{w}} \cdot \frac{\mu_{w}}{S \mu+\mu_{w}} .
$$

In this study, $P_{b 1}$ is the same for both queuing disciplines, whereas probability $P_{b 2}$ is different. Simulation results have confirmed that $P_{b 1}$ values are almost the same in both cases (see Section VII and in particular Fig. 8).

The efficiency of the LUI queuing discipline as regards the FIFO one depends on the following parameters: the mobility parameter $\alpha$, the number of channels per cell $S$, and the traffic intensity per cell due to new call arrivals $\lambda T_{m}$. Various simulations have demonstrated a slight dependence of this efficiency on $\alpha$ and $\lambda T_{m}$, whereas a greater sensitivity to parameter $S$ is expected.

Note that a recursive approach is necessary to compute $P_{b 1}$ and $P_{b 2}$ (for both queuing disciplines) because $\lambda_{h}$ is related to $P_{b 1}$ and $P_{b 2}$ through (17). We start the iterative method with $n_{h}$ for $P_{b 1}=P_{b 2}=0$ (this is the maximum value of $n_{h}$ : i.e., $n_{h}=4 /(3 \alpha) ; n_{h}$ decreases for increasing values of $P_{b 1}$ or $\left.P_{b 2}\right)$. With such a value of $n_{h}, \mu$, and $P_{n}$ for $n=0,1, \cdots$ are computed according to formulas (30), (32), and (31). These values are used to compute $P_{b 1}$ and $P_{b 2}$ (for a queuing discipline) and then the new value of $n_{h}$. This value is averaged with the previous one [i.e., $4 /(3 \alpha)$ at the first step]. A new iteration starts with this average value of $n_{h}$. The iterative method is stopped when the relative difference between the $n_{h}$ values computed in two subsequent steps is 


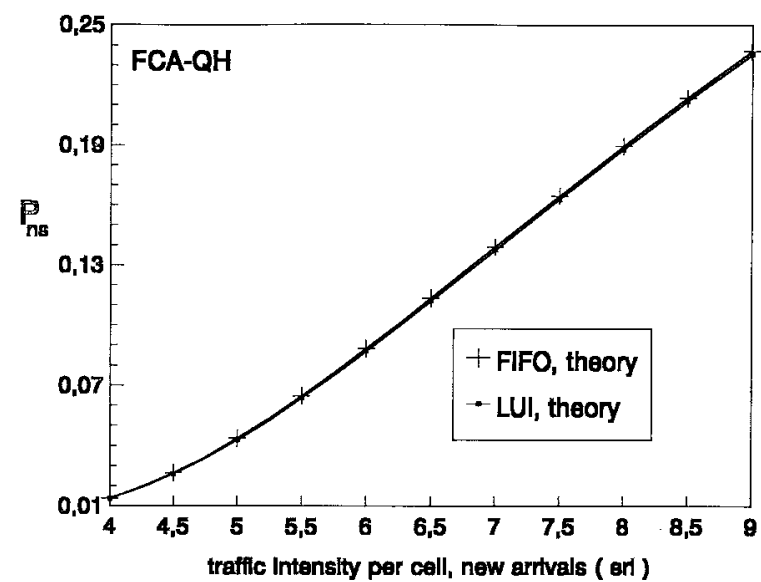

Fig. 5. Theoretic comparison in terms of $P_{n s}$ for FCA-QH between FIFO and LUI queuing disciplines (IRIDIUM case).

below a given threshold (e.g., 10 $\left.10^{-3}\right)$. Finally, we can derive $P_{n s}$ from (22).

Fig. 5 shows the analytical results obtained for FCA-QH with both FIFO and LUI schemes in the IRIDIUM mobility case. These analytical results show that the LUI strategy gives rise to a value of $P_{n s}$ less than the FIFO one, but the performance differences of these two queuing schemes are extremely reduced in terms of $P_{n s}$.

\section{Simulation RESUlts}

The following assumptions have been made for the simulations.

- The call arrival process is Poisson independent from cell to cell with average call arrival rate per cell equal to $\lambda$.

- The call duration is exponentially distributed with average value equal to $T_{m}=3 \mathrm{~min}$.

- The reuse distance is $D=\sqrt{21} R$; then, the FCA cluster is formed by seven cells.

- The simulated cellular network is parallelogram shaped and folded onto itself with seven cells per side.

- A number of 70 channels is available to the system. Then, ten channels are permanently allocated to each cell with FCA.

- The IRIDIUM mobility case is considered $(\alpha \approx 0.27)$.

- An infinite queue capacity is assumed.

The comparison between simulations and analytical predictions for FCA-QH with FIFO and LUI queuing disciplines are, respectively, shown in Figs. 6 and 7 in terms of parameter $P_{n s}$. We may note that the theoretic approaches for both disciplines give a conservative estimate of the blocking performance obtained by simulations. This difference is exclusively due to the approximations of the analysis: the fitted exponential distribution for the maximum queuing time, the Poisson arrival process for handovers, and the fitted exponential distribution for the channel holding time. However, due to the good fitting between the exponential distributions and the actual ones, we have that the approximation of these results is mainly due to the assumption on the Poisson arrival process for handovers. Since the handover traffic is smooth, it gives a lower blocking probability than a Poisson traffic at a parity of mean arrival

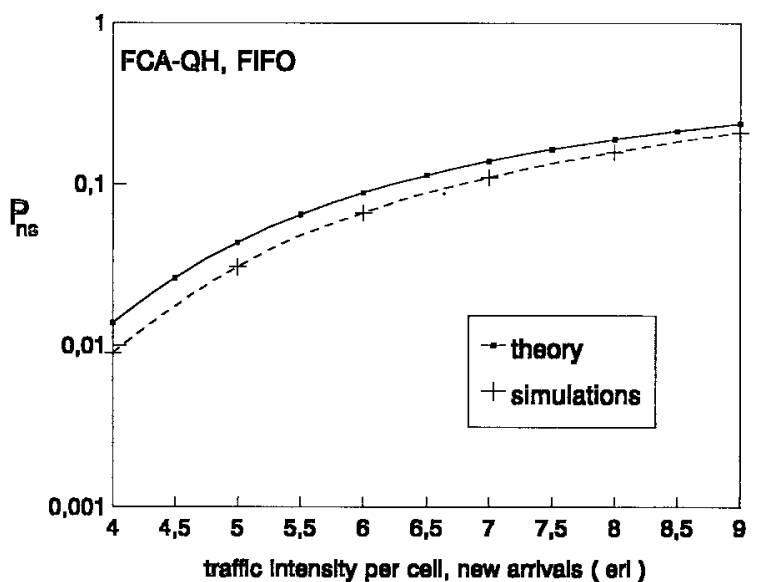

Fig. 6. FCA-QH with FIFO queuing discipline: comparison between simulations and analytical predictions (IRIDIUM case).

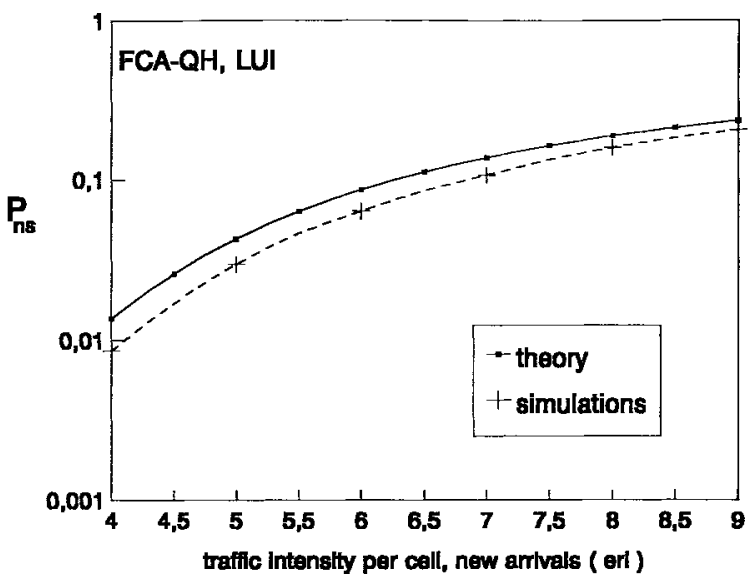

Fig. 7. FCA-QH with LUI queuing discipline: comparison between simulations and analytical predictions (IRIDIUM case).

rate, service time distribution, and number of servers [23]. This consideration explains the upper bound obtained by theory.

Figs. 8-10 show simulation results in terms of $P_{b 1}, P_{b 2}$, and $P_{n s}$, respectively. In these graphs, the behavior of FCA with no prioritization scheme (NPS) has been also presented. The following considerations arise.

- FCA-QH strategies (both FIFO and LUI) have values of $P_{b 1}$ greater than FCA NPS, but values of $P_{b 2}$ far less than FCA NPS. The advantage of the QH approach (with whatsoever queuing discipline) with respect to NPS is more evident in terms of $P_{n s}$ : the QH solution significantly reduces $P_{n s}$ with respect to the scheme without any prioritization (NPS). This result is very important for future high-mobility systems (such as LEO-MSS's under examination), where handover requests will be extremely frequent during call lifetime (e.g., in Section III referring to the IRIDIUM system, we have shown that when $P_{b 1}=P_{b 2}=0$, there are, on average, about 4.82 handover requests during a call which has an expected duration of $3 \mathrm{~min}$ ).

- Figs. 8-10 permit comparing the performance of FCA$\mathrm{QH}$ with FIFO and LUI queuing disciplines. It is 


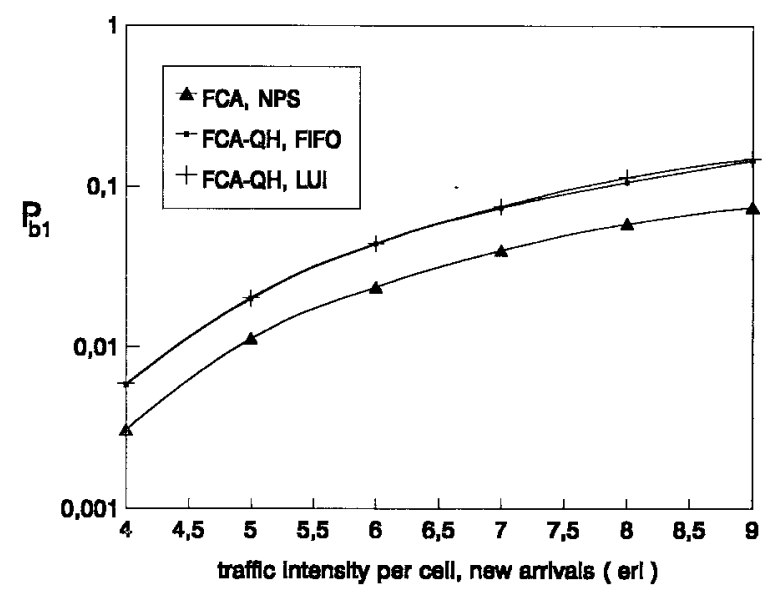

Fig. 8. $\quad P_{b 1}$ performance for FCA NPS, FCA-QH FIFO, and FCA-QH LUI (IRIDIUM case).

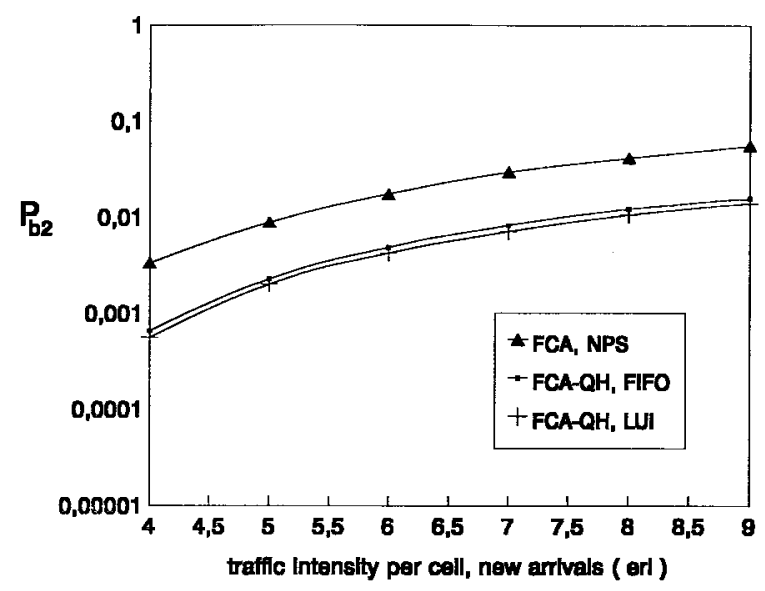

Fig. 9. $\quad P_{b 2}$ performance for FCA NPS, FCA-QH FIFO, and FCA-QH LUI (IRIDIUM case).

important to stress that the LUI performance in terms of $P_{b 2}$ is the best possible among all the queuing disciplines. Simulations have verified that the LUI queuing discipline permits to reduce $P_{b 2}$ with respect to the FIFO one (see Fig. 9), but this difference is very little, whereas LUI and FIFO solutions have practically the same performance in terms of $P_{b 1}$ (see Fig. 8). Finally, the $P_{n s}$ performance of the FIFO scheme is near to the LUI one (see Fig. 10).

Therefore, we have that the ideal LUI scheme permits achieving a very slight performance improvement as regards the FIFO one. In addition to this, we have shown that an implementation of the LUI scheme would entail a greater complexity than the FIFO solution. In conclusion, the comparison between the simple FIFO scheme and the LUI one has permitted to validate the goodness of the FIFO approach in LEO-MSS's.

\section{CONCLUSIONS}

In this paper, we have investigated resource management strategies in LEO-MSS's. A suitable mobility model has been proposed. An FCA technique has been assumed. However, handover requests which cannot be immediately accomplished may be queued for a maximum time. Two queuing disciplines

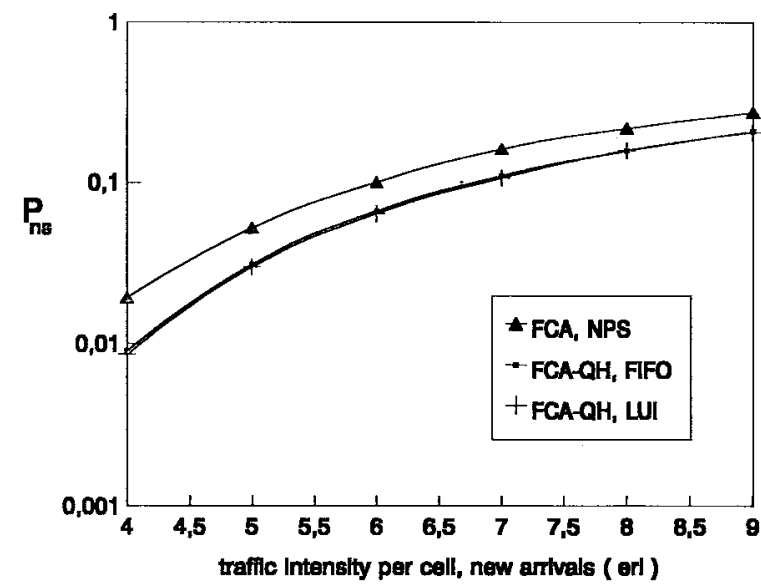

Fig. 10. $P_{n s}$ performance for FCA NPS, FCA-QH FIFO, and FCA-QH LUI (IRIDIUM case).

have been investigated: the FIFO scheme and the idealized LUI technique.

Analytical and simulation results have shown that the FIFO policy attains good results very close to the bound given by the LUI technique. Therefore, the FIFO solution has to be preferred to the LUI one which would require a greater implementation complexity.

\section{ACKNOWLEDGMENT}

The authors would like to thank the unknown reviewers for providing useful comments to improve the quality of this paper.

\section{REFERENCES}

[1] E. Del Re, "A coordinated European effort for the definition of a satellite integrated environment for future mobile communications," IEEE Commun. Mag., vol. 34, pp. 98-104, Feb. 1996.

[2] ___ "Satellite system integrated with the terrestrial cellular network for mobile communications," ESA Tech. Rep., ESA STR-228, Aug. 1989.

[3] _ "Objectives and research activities of COST 227 project-Integrated space/terrestrial mobile networks," in RACE Mobile Telecommun. Workshop, Amsterdam, The Netherlands, May 17-19, 1994, pp. 590-597.

[4] G. Maral, J.-J. De Ridder, B. G. Evans, and M. Richharia, "Low earth orbit satellite systems for communications," Int. J. Sat. Commun., vol. 9 , pp. 209-225, 1991.

[5] J. L. Grubb, "IRIDIUM overview," IEEE Commun. Mag., vol. 29, no. 11, Nov. 1991

[6] S. Tekinay and B. Jabbari, "Handover and channel assignments in mobile cellular networks," IEEE Commun. Mag., vol. 29, pp. 42-46, Nov. 1991.

[7] _ _A measurement-based prioritization scheme for handovers in mobile cellular networks," IEEE J. Select. Areas Commun., vol. 10, pp. 1343-1350, Oct. 1992

[8] D. Hong and S. S. Rappaport, "Traffic model and performance analysis for cellular mobile radio telephone systems with prioritized and nonprioritized handoff procedures," IEEE Trans. Veh. Technol., vol. VT-35, pp. 77-92, Aug. 1986.

[9] S. S. Rappaport, "Blocking, hand-off and traffic performance for cellular communication systems with mixed platforms," Proc. Inst. Elect. Eng., vol. 140, pp. 389-401, Oct. 1993.

[10] ITU-T Recommendation E.771, "Network grade of service parameters and target values for circuit-switched land mobile services."

[11] L. J. Cimini, G. J. Foschini, C.-L. I, and Z. Miljanic, "Call blocking performance for dynamic channel allocation in microcells," IEEE Trans. Commun., vol. 42, pp. 2600-2607, Aug. 1994.

[12] T.-P. Chu and S. Rappaport, "Overlapping coverage and channel rearrangement in microcellular communication systems," in Proc. IEEE 
GLOBECOM '94, San Francisco, CA, Nov. 28-Dec. 2, 1994, pp. 1674-1678.

[13] E. Del Re, R. Fantacci, and G. Giambene, "An efficient technique for dynamically allocating channels in satellite cellular networks," in Proc. IEEE GLOBECOM '95, Singapore, Nov. 13-17, 1995, pp. 1624-1628.

[14] _ "Efficient dynamic channel allocation techniques with handover queuing for mobile satellite networks," IEEE J. Select. Areas Commun., vol. 13, pp. 397-405, Feb. 1995.

[15] Y.-B. Lin, L.-F. Chang, and A. Noerpel, "Modeling hierarchical microcell/macrocell PCS architecture," in IEEE ICC'95, Seattle, WA, June 1995 , pp. 405-409.

[16] B. Jabbari, G. Colombo, A. Nakajima, and J. Kulkarmi "Network issues for wireless communications," IEEE Commun. Mag., vol. 33, pp. 88-98, Jan. 1995.

[17] Y.-B. Lin, S. Mohan, and A. Noerpel, "Queuing priority channel assignment strategies for PCS hand-off and initial access," IEEE Trans. Veh. Technol., vol. 43, pp. 704-712, Aug. 1994.

[18] _ , "PCS channel assignment strategies for hand-off and initial access," IEEE Personal Commun., pp. 47-56, 1994.

[19] _ _ "Queueing channel assignment strategies for PCS hand-off and initial access," in Proc. ICUPC'94, San Diego, CA, Sept. 27-Oct. 1, 1994.

[20] W. Zhao, R. Tafazolli, and B. G. Evans, "A UT positioning approach for dynamic satellite constellations," in Proc. 4th IMSC'95, Ottawa, Canada, June 6-8, 1995, pp. 251-258.

[21] SAINT Project (RACE 2117), "Functional models for location monitoring," WP 4100, June 1995.

[22] V. H. MacDonald, "The cellular concept," Bell Syst. Tech. J., vol. 58, pp. 15-41, Jan. 1979.

[23] A. A. Fredericks, "Congestion in blocking systems-A simple approximation technique," Bell Syst. Tech. J., vol. 59, no. 6, pp. 805-825, July-Aug. 1980.

Enrico Del Re (M'78-SM'84) was born in Florence, Italy, in 1947. He received the Dr.Ing. degree in electronics engineering from the University of Pisa, Pisa, Italy, in 1971.

Until 1975, he worked in public administration and private firms, where he was involved in the analysis and design of the telecommunication and air traffic control equipment and space systems. Since 1975, he has been with the Department of Electronics Engineering, University of Florence, Florence, Italy, first as a Research Assistant and then as an Associate Professor. Since 1986, he has been a Professor at the University of Florence. During the academic year 1987-1988, he was on leave from the University of Florence for a nine-month period of research at the European Space Research and Technology Center of the European Space Agency, The Netherlands. He is the Head of the Digitial Signal Processing and Telematics Laboratory (LENST), Department of Electronic Engineering, University of Florence. $\mathrm{He}$ also teaches a course in digital signal processing. His main research interests are digital signal processing, digital transmission techniques, and communication networks, on which he has published more than 150 papers in international journals and conferences. He is the coeditor of Satellite Integrated Communications Networks (North-Holland, 1988) and a coauthor of Data Compression and Error Control Techniques with Applications (New York: Academic, 1985).

Dr. Del Re has been the Chairman of the European Project COST 227 "Integrated space/terrestrial mobile networks" and is now Chairman of the COST 252 Project "Evolution of satellite personal communications from second to future generation systems." He received the 1988/89 premium from the IEE (U.K.) for the paper "Multicarrier demodulator for digital satellite communication systems." He has been included in Who's Who in the World. $\mathrm{He}$ is a member of the AEI and European Association for Signal Processing (EURASIP).

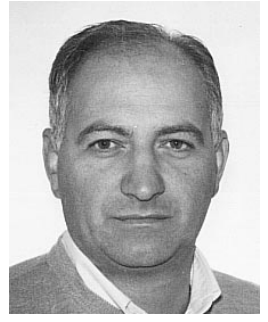

Romano Fantacci (S'82-M'87-SM'91) was born in Pistoia, Italy. He received the Dr.Ing. degree in electronics engineering in 1982 and the Ph.D. degree in telecommunication engineering in 1987 , both from the University of Florence, Florence, Italy.

In 1982, he joined the Electronics Engineering Department, University of Florence, as a Researcher in Computer Communications, where he is now an Associate Professor of Telecommunication Networks. During the first stage of his research activity, he worked on satellite communication systems. In particular, he has been involved in several European Space Agency (ESA) and INTELSAT advanced research projects. His present research interests involve digital communications, computer communications, queueing theory, fast packet switching, and mobile communication networks. He is the author of works that have been published in several journals in communication science.

Dr. Fantacci has been involved in several European (RACE) and nationwide research projects sponsored by the Italian National Council of Research (C.N.R.) and Italian Minister of Education and Research (MURST).

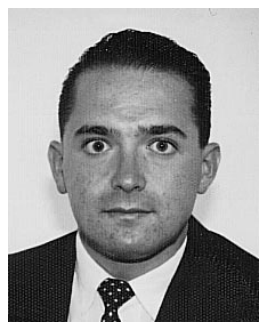

Giovanni Giambene (S'94-M'97) received the Dr.Ing. degree in electronics in 1993 and the Ph.D. degree in telecommunications and informatics in 1997, both from the University of Florence, Florence, Italy.

Since 1994, he has been with the Electronic Engineering Department, University of Florence, as a Research Assistant. His research interests include mobile cellular communication networks, personal communication services, channel allocation techniques, and queuing theory.

Dr. Giambene was the Technical External Secretary of the European Community Project COST 227 "Integrated space/terrestrial mobile networks." $\mathrm{He}$ also contributed to the "resource management" activity of Working Group 3000 within the RACE Project called "Satellite integration in the future mobile network" (SAINT, RACE 2117). 